\title{
Paxillin promotes the migration and angiogenesis of HUVECs and affects angiogenesis in the mouse cornea
}

\author{
WAN-JU YANG ${ }^{1,2}$, JIANG-BO YAN ${ }^{1}$, LI ZHANG $^{2}$, FANG ZHAO $^{2}$, ZHONG-MING MEI $^{2 *}$, \\ YAN-NING YANG $^{1}$, YI XIANG ${ }^{2}$ and YI-QIAO XING ${ }^{1}$ \\ ${ }^{1}$ Eye Center, Renmin Hospital of Wuhan University, Wuhan, Hubei 430060; ${ }^{2}$ Department of Ophthalmology, \\ The Central Hospital of Wuhan, Tongji Medical College of Huazhong University of Science and Technology, \\ Wuhan, Hubei 430014, P.R. China
}

Received August 9, 2018; Accepted August 7, 2019

DOI: $10.3892 /$ etm.2020.8751

\begin{abstract}
Neonatal vascular ophthalmopathy is a refractory ophthalmologic disease, and is a major cause of blindness. Occurrence of neonatal vascular ophthalmopathy may be associated with Paxillin, a cellular adhesion molecule which promotes the migration of endothelial cells and angiogenesis. To explore the role of PXN in corneal angiogenesis, human umbilical vein endothelial cells were divided into five groups: i) Control group; ii) Empty vector-transfected control group; iii) PXN knockdown group (shPXN group); iv) PXN-negative control (NC) group; and v) PXN over-expressed group (overExp group). PXN protein levels, migration and tube formation were assessed in the different experimental groups. Mice were divided into four groups: i) Control; ii) Model; iii) shPXN; and iv) overExp groups. Tube formation was significantly increased in the overExp group compared with the empty vector-transfected control group $(\mathrm{P}<0.01)$. Tube formation was significantly decreased in the shPXN group compared with the PXN-NC group $(\mathrm{P}<0.01)$. In mice, blood corpuscles were significantly decreased in the shPXN group. PXN promoted the migration of endothelial cells and corneal angiogenesis. The results of the present study suggest a role for
\end{abstract}

Correspondence to: Dr Yi Xiang, Department of Ophthalmology, The Central Hospital of Wuhan, Tongji Medical College of Huazhong University of Science and Technology, 26 Shengli Avenue, Wuhan, Hubei 430014, P.R. China

E-mail: 3528596168@qq.com

Dr Yi-Qiao Xing, Eye Center, Renmin Hospital of Wuhan University, 99 Zhang Zhidong Road, Wuhan, Hubei 430060, P.R. China

E-mail: xing_yiqiao@aliyun.com

"Deceased

Abbreviations: PXN, paxillin; HUVEC, human umbilical vein endothelial cells

Key words: Paxillin, corneal angiogenesis, HUVECs, migration, tube formation
PXN in corneal angiogenesis and provide a theoretical basis and potential target for the treatment of corneal angiogenesis.

\section{Introduction}

The cornea is a transparent, avascular structure that lacks lymphatic vessels. It is the first important refractive medium in the eye and factors such as immune disease, infection and trauma can lead to neovascularization from the corneal limbus to the cornea. The clinical term for the formation of a new capillary network in the cornea is corneal neovascularization. Neovascular disease is an intractable disease in ophthalmology, and is the major cause of blindness in a number of eye diseases such as diabetic retinopathy, retinopathy of prematurity and occlusion of the central or branch of the retina (1-4). Retinal neovascularization is a complicated process. First, vasodilation occurs, and permeability is increased. Subsequently, basal membrane enzymes degrade the vascular wall, while vascular basement membrane enzymes aid in endothelial cell chemotaxis and migration. Mitosis in peripheral cells promotes the formation of a new vascular cavity in the intraocular vascular endothelial cells caused by ocular exudation. This gap increases bleeding and causes hyperplasia and pathological changes, which leads to irreversible damage to the structure and function of the eye, ultimately resulting in a serious decline in vision and potentially blindness. Keratitis is a common eye disorder that often leads to the formation of new blood vessels $(5,6)$. Paxillin (PXN) is a focal adhesion protein, and is comprised of an LD motif (containing the consensus peptide sequence LDXLLXXL), a conserved cysteine-rich domain comprising of $\sim 60$ amino acids with seven conserved cysteine residues and a histidine residue (LIM domain), Src homology 2 (SH2) domain and Src homology 3 (SH3) domains (7). A previous study demonstrated that PXN also serves an important role in corneal neovascularization (8). Vascular endothelial growth factor (VEGF) is an important pro-angiogenic factor required during the process of angiogenesis, and is a highly specific vascular endothelial cell mitogen, which has been previously reported to be regulated by PXN (9). The role of the PXN signaling pathway in the process of corneal neovascularization induced by inflammation is not clear. In the present study, human umbilical vein endothelial cells (HUVECs) and mouse corneal 
neovascularization models were used to investigate changes in the PXN signaling pathway during corneal neovascularization induced by inflammation. The results of the present study shed light on the role of PXN in corneal neovascularization and provide a theoretical basis and potential target for the treatment of corneal neovascularization.

\section{Materials and methods}

HUVEC cell culture and grouping. HUVECs were cryopreserved at the Department of Ophthalmology, Wuhan University (Wuhan, China), and seeded into poly-L-lysine-coated flasks and maintained in endothelial complete medium supplemented with $5 \% \mathrm{FBS}, 1 \%$ penicillin/streptomycin and $1 \%$ endothelial cell growth supplement (All from ScienCell Research Laboratories, Inc.). The cells were maintained at $37^{\circ} \mathrm{C}$ in a humidified incubator with $5 \% \mathrm{CO}_{2}$, and the medium was replaced every 2-3 days until the cells were confluent. Cells were harvested with $0.05 \%$ trypsin-ethylene glycol tetra-acetic acid solution (Wuhan Boster Biological Technology, Ltd.) and were further cultured in the poly-L-lysine-coated flasks for use in subsequent experiments. Cell at passage five were used, when they lost their cobblestone like appearance.

HUVECs were divided into 5 groups: Control group, empty vector-transfected control group (EV group), PXN knockdown group (shPXN group), PXN-negative control (NC) group (NC group) and PXN over-expressed group (overExp group).

Lentivirus preparation. The positive-sense strand and antisense strands of the oligonucleotide fragments of the PXN short hairpin (sh)RNA (forward, 5'-GATCCAAAGTTTCGG GATCCAATCTCTTCAAGAGAGAGATTGGATCCCGAA ACTTTTTTTTTCTCGAGG-3' and reverse, 5'-AGCTCC TCGAGAAAAAAAAAGTTTCGGGATCCAATCTCTCTC TTGAAGAGATTGGATCCCGAAACTTTG-3'; synthesized by Suzhou GenePharma, Co., Ltd.) were annealed. They were dissolved in double-steamed water, mixed in equal molar amounts, heated at $90^{\circ} \mathrm{C}$ for $3 \mathrm{~min}$, and slowly cooled to $37^{\circ} \mathrm{C}$ to form double-stranded oligonucleotides. The synthesized shRNA was inserted into the shRNA expression vector pSilencer 2.1-U6neo (Suzhou GenePharma, Co., Ltd.) and digested with BamHI and HindIII restriction enzymes to construct the shRNA recombinant plasmid for PXN.

Ad-PXN was synthesized and identified by Hanbio Biotechnology Co., Ltd. HUVECs in the logarithmic growth phase and in a good growth state were digested with $0.25 \%$ trypsin and cultured in growth medium $(10 \% \mathrm{FBS}+1 \%$ double antibody). A cell suspension was prepared and plated onto 6-well plates at a density of $5 \times 10^{5}$ cells/well. Cells were cultured in saturated humidity at $37^{\circ} \mathrm{C}$ with $5 \% \mathrm{CO}_{2}$ until they reached $70 \%$ confluence. A total of $25 \mu \mathrm{l}$ Ad-PXN was added to each well and cells were further cultured for $24 \mathrm{~h}$.

$R N A$ extraction and reverse transcription-quantitative $(R T-q) P C R$. Total RNA was extracted from corneal tissue or HUVECs using TRIzol ${ }^{\circledR}$ reagent (Thermo Fisher Scientific, Inc.). For each sample, $1 \mu \mathrm{g}$ RNA was reverse transcribed using PrimeScript ${ }^{\circledR}$ RT reagent kit with gDNA Eraser (Takara Bio, Inc.) according to manufacturer's protocol. The following temperature protocol was used: DNA extension at $42^{\circ} \mathrm{C}$ for $60 \mathrm{~min}$ followed by inactivation at $70^{\circ} \mathrm{C}$ for $15 \mathrm{~min}$ and a holding temperature of $16^{\circ} \mathrm{C}$, to obtain first-strand cDNA. Expression levels of target genes Ras, FAK, Src, Rho, Rac and Cdc42 were analyzed using RT-qPCR. Primer premier software (v5.0; Premier Biosoft International) was used to design the fluorescent primers (Table I). The reaction contained $10 \mu \mathrm{l}$ 2x SYBR ${ }^{\circledR}$ Premix Ex Taq ${ }^{\mathrm{TM}}$ (Takara Bio, Inc.), $0.50 \mu \mathrm{mol} / 1$ of each primer and $0.2 \pm 0.02 \mu \mathrm{g}$ cDNA template, and made to a final volume of $20 \mu \mathrm{l}$ using nucleotide free water. The thermocycling conditions were: Pre-denaturation at $95^{\circ} \mathrm{C}$ for $30 \mathrm{sec}$; followed by 39 cycles of denaturation at $95^{\circ} \mathrm{C}$ for $5 \mathrm{sec}$, annealing at $56^{\circ} \mathrm{C}$ for $10 \mathrm{sec}$ and elongation at $72^{\circ} \mathrm{C}$ for $25 \mathrm{sec}$. Gene expression was normalized to that of $\beta$-actin and quantified using the $2^{-\Delta \Delta \mathrm{Cq}}$ method (10).

Western blot analysis. Western blot analysis was performed to determine the protein expression levels of Rho, Rac, Cdc42, SRC and FAK. The cells were homogenized with lysis buffer (cat. no. AB100086, Bio-swamp Life Science) and centrifuged at $12,000 \mathrm{x} \mathrm{g}$ at $4^{\circ} \mathrm{C}$ for $20 \mathrm{~min}$. The protein concentration was determined using a bicinchoninic acid assay kit (Bio-swamp Life Science). Equal quantities of protein $(30 \mu \mathrm{g})$ were resolved on a $10 \%$ gel using SDS-PAGE and subsequently transferred to a PVDF membrane (EMD Millipore; Merck KGaA). The membranes were blocked for $2 \mathrm{~h}$ at room temperature with 5\% skimmed milk in TBS-Tween 20 (20 mmol/1 Tris, $500 \mathrm{mmol} / 1 \mathrm{NaCl}$, and $0.05 \%$ Tween 20 ). Subsequently, the membrane was incubated with one of the following primary antibodies (all from Abcam) overnight at $4^{\circ} \mathrm{C}$ : Rabbit anti-BMP-2 antibody (cat. no. ab14933; 1:1,000), rabbit anti-VEGF antibody (cat. no. ab32152; 1:4,000) and human anti-CtII antibody (cat. no. ab159157; 1:2,000). Anti-GAPDH antibody (cat. no. ab181602; 1:10,000) was used as the loading control. Following incubation with the primary antibody, the membranes were washed with TBS and incubated in goat anti-rabbit secondary antibody (cat. no. PAB160009; Bioswamp Life Science; 1:10,000) for $2 \mathrm{~h}$ at room temperature. The signals were visualized using enhanced chemiluminescent substrate buffer (EMD Millipore). Membranes were scanned using Tanon 5200 Chemiluminescent Imaging System (Tanon Science and Technology Co., Ltd.).

Transwell migration assay. Prior to the experiment, cells were cultured in serum-free medium, and cultured for $24 \mathrm{~h}$. PBS was added to 24-well plates with the Transwell inserts (Corning, Inc.) for $5 \mathrm{~min}$ to soak the chambers, and the cells were washed with serum-free medium. A $0.5 \mathrm{ml}$ cell suspension containing $1 \times 10^{5}$ cells $/ \mathrm{ml}$ in $1 \% \mathrm{FBS}$ was added to the upper chamber, and $0.75 \mathrm{ml}$ DMEM with $10 \%$ FBS was added to the lower chamber. Cells were cultured at $37^{\circ} \mathrm{C}$ with $5 \% \mathrm{CO}_{2}$ for $48 \mathrm{~h}$. Subsequently, the media was removed and $1 \mathrm{ml}$ $4 \%$ formaldehyde solution was added to fix the cells at $25^{\circ} \mathrm{C}$ for $10 \mathrm{~min}$. Following fixing, the cells were washed with PBS, and stained with $1 \mathrm{ml} 0.5 \%$ crystal violet solution for $30 \mathrm{~min}$ at $25^{\circ} \mathrm{C}$. Cells which had not migrated were gently removed using a cotton swab once the chambers had dried. The number of migrated cells were observed and counted at a magnification, x200 under a inverted fluorescence microscope (DMIL LED; Leica Microsystems GmbH). The Transwell migration assays were performed in triplicate. 
Table I. Primer sequences.

\begin{tabular}{|c|c|c|}
\hline Primer & Sequence & Size, base pairs \\
\hline Rho-F & 5'-TCACGCTATCATGGGTGTGG-3' & 226 \\
\hline Rho-R & 5'-CAGCTGCCCATAGCAGAAGA-3' & \\
\hline Rac-F & 5'-CATTCCAGACTCACGACC-3' & 128 \\
\hline Rac-R & 5'-CACAATCTCCGCACCATA-3' & \\
\hline Cdc42-F & 5'-TGTTTCTCAGTGGTCTCTCCA-3' & 287 \\
\hline Cdc42-R & 5'-GCAGCCAATATTGCTTCATCA-3' & \\
\hline Src-F & 5'-CGCCTCACTACCGTATGTCC-3' & 250 \\
\hline Src-R & 5'-CCAGTTTCTCGTGCCTCAGT-3' & \\
\hline FAK-F & 5'-TGTCCTCCCACССТСТАC-3' & 138 \\
\hline FAK-R & 5'-CCTCATCCGTTCTTCTTG-3' & \\
\hline PXN-F & 5'-CTTGACCGGCTGTTACTG-3' & 180 \\
\hline PXN-R & 5'-TCGCTTTGGCTTCTCTTT-3' & \\
\hline$\beta$-Actin-F & 5'-ACACTGTGCCCATCTACG-3' & 153 \\
\hline$\beta$-Actin-R & 5'-TGTCACGCACGATTTCC-3' & \\
\hline
\end{tabular}

F, forward; R, reverse; RHO, Rho-GTPase; Rac, AKT serine/threonine kinase 1; Cdc42, cell division control protein 42; Src, SRC proto-oncogene, non-receptor tyrosine kinase; FAK, focal adhesion kinase; PXN, rpaxillin.

Experimental animals. Mice (age, 2 months) were obtained from Hubei Provincial Center for Disease Control and Prevention. A total of 24 female mice, weighing 20-22 g, were used. To ensure a healthy corneal surface, all eyes were carefully examined using an operating microscope (Carl Zeiss AG) prior to any experimental procedures.

Mice were placed under general anesthesia through an intramuscular injection of a ketamine mixture (ketamine $60 \mathrm{mg} / \mathrm{kg}+$ xylazine $8 \mathrm{mg} / \mathrm{kg}$ ), supplemented with topical anesthesia with $0.1 \%$ proparacaine hydrochloride. A $3 \mathrm{~mm}$ diameter filter paper disc was soaked in $1.0 \mathrm{M} \mathrm{NaOH}$ for $1 \mathrm{~min}$ and used to cause a corneal burn. The disc was applied to the cornea, 1-2 $\mathrm{mm}$ from the limbus and then removed after $2 \mathrm{~min}$. The corneal surface was rinsed thoroughly with $10 \mathrm{ml} 0.9 \%$ $\mathrm{NaCl}$ solution. To ensure maximum reproducibility, all alkali burns were performed by the same investigator. Knockdown and overexpression model mice were established via a tail vein injection of lentivirus with a titer of $1 \times 10^{8} \mathrm{TU} / \mathrm{ml}$.

According to the criteria of animal pain judged by the Welfare and Ethical Reference of Experimental Animal Center of Zhejiang University, mild pain manifested as a weight loss of $\sim 5 \%$, only $50-75 \%$ of normal intake within $72 \mathrm{~h}$, a small amount of erect hair, normal interactions with other mice, and normal sensitivity to external stimuli $(11,12)$.

In the present study, eye inflammation was observed microscopically on the 14th day after modeling. The physiological habits of the animals were continuously monitored. Although blood vessels were observed in the eyes, the mice in each group showed no abnormal symptoms, and the diet, body weight and behavior were normal. On the 21st day, two mice presented few erect hairs, exhibited normal interactions with other mice and remained sensitive to external stimuli, although 2 mice were found scratching their eyes with their front paws. If the mice suffered from mild pain on the 21 st day according to the aforementioned criteria of animal pain, then the experiment was stopped immediately. The mice were sacrificed at day 21 with an overdose of sodium pentobarbital (100 mg/kg, intraperitoneally), and samples were collected.

All experimental protocols were approved by the Institutional Animal Care and Use Committee of Tongji Medical of Huazhong University of Science and Technology and Animal Experimental Ethical Inspection of Laboratory Animal Centre, Huazhong Agriculture University; approval no. HZAUMO-2017-039.

Cell tube formation assay. Prior to the experiment, all products that were exposed to the Matrigel matrix (BD Biosciences) adhesive were placed in a $-20^{\circ} \mathrm{C}$ refrigerator for $6 \mathrm{~h}$. Subsequently, the Matrigel matrix glue was placed in a $4^{\circ} \mathrm{C}$ refrigerator for $24 \mathrm{~h}$. When the matrix was in a liquid form, $60 \mu 1$ Matrigel was added per well into 96-well plates, and placed at $37^{\circ} \mathrm{C}$ for $1 \mathrm{~h}$. The formation of HUVEC capillary-like structures on a basement membrane matrix were used to investigate the angiogenic activity of Paxillin. HUVECs were then seeded onto the Matrigel bed $\left(1.5 \times 10^{4}\right.$ cells/well $)$ and cultured for $6 \mathrm{~h}$. Tube formation was imaged and the tube lengths were quantified using the Image-Pro ${ }^{\circledR}$ Plus software (v6.0; Media Cybernetics, Inc.).

VEGF expression was detected by immunohistochemistry. Following removal of the corneal tissue, the tissue was fixed in $4 \%$ polyformaldehyde for $24 \mathrm{~h}$, the paraffin embedded sections were sliced into 4-5 $\mu \mathrm{m}$ samples. Subsequently, the samples were dewaxed using dimethylbenzene, after which the samples were incubated on a compound digestion solution for enzyme repair for $30 \mathrm{~min}$ at $25^{\circ} \mathrm{C}$. Samples were flushed with PBS and $3 \%$ hydrogen peroxide was added to block peroxidase activity. Samples were blocked in goat serum (cat. no. SL038, Beijing Solarbio Science \& Technology Co., Ltd.) at $37^{\circ} \mathrm{C}$ for 


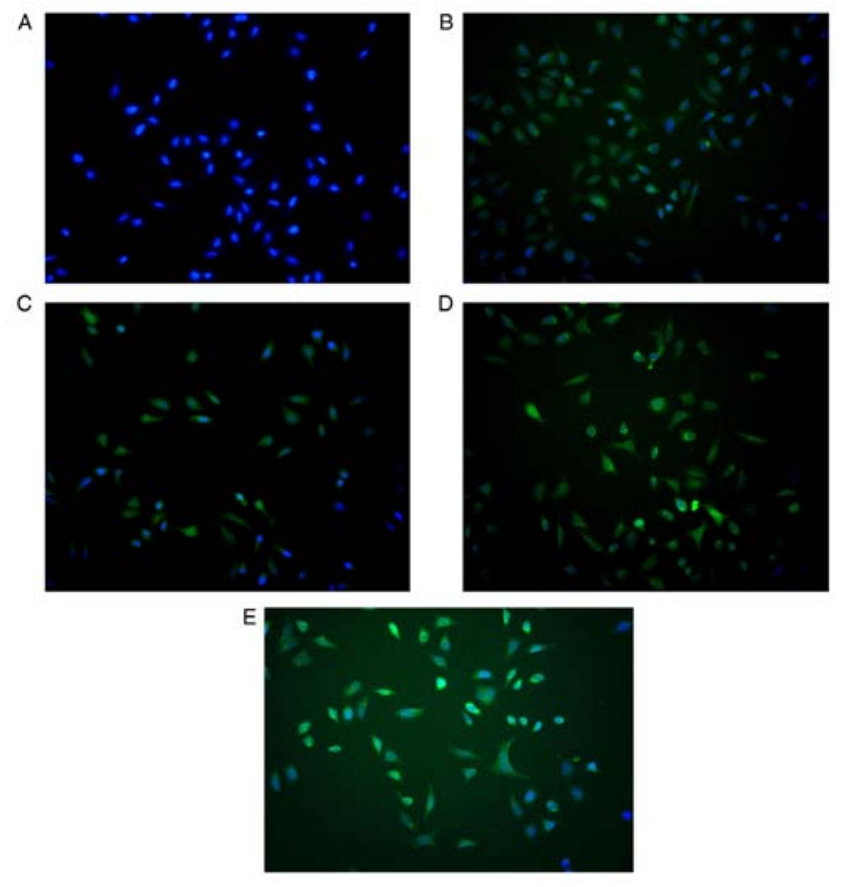

Figure 1. Lentiviral vector expression cassette allows for expression of PXN in transduced cells. Fluorescence microscopy of (A) the control group, (B) empty vector-transfected control group, (C) PXN knockdown group, (D) PXN-negative control group and (E) PXN overexpression group. PXN, paxillin.

$30 \mathrm{~min}$, and incubated with anti-VEGF antibody (1:100; cat. no. PAB30096, Bio-swamp Life Science), at $4^{\circ} \mathrm{C}$ overnight. Subsequently, the samples were biotinylated by incubating the samples with biotin $(4 \mathrm{mg} / \mathrm{ml}$; cat. no. CYB167077-FTK, ZSGB-BIO; OriGene Technologies, Inc.) at room temperature for $30 \mathrm{~min}$ to mark the goat anti-rabbit and horseradish enzyme labelled chain enzyme working fluid (1:200; cat. no. PAB160022; Bio-swamp Life Science), and 3,3'-diaminobenzidine color reagent (cat. no. PAB180021; Bio-swamp Life Science) was added to the enzyme. Samples were observed under a microscope, and brown or yellow staining indicated positive expression. Samples were counterstained with hematoxylin for $3 \mathrm{~min}$ at $4^{\circ} \mathrm{C}$. Subsequently an alcohol series was used to dehydrate the solution and the slides were sealed with neutral gum.

Statistical analysis. All data are expressed as the mean \pm standard deviation. Statistical analysis was performed in SPSS version 22.0 (IBM, Corp.) and groups were compared using a one-way ANOVA with a post-hoc Duncan's test. $\mathrm{P}<0.05$ was considered to indicate a statistically significant difference.

\section{Results}

Expression of PXN in HUVECs. The lentiviral vector expression cassette allowed for the expression of PXN in transduced cells (Fig. 1). There were no notable differences in fluorescence intensity between the EV, shPXN, NC, and overExp groups.

Involvement of PXN pathway in angiogenesis. The protein expression levels of PXN were detected by western blotting.

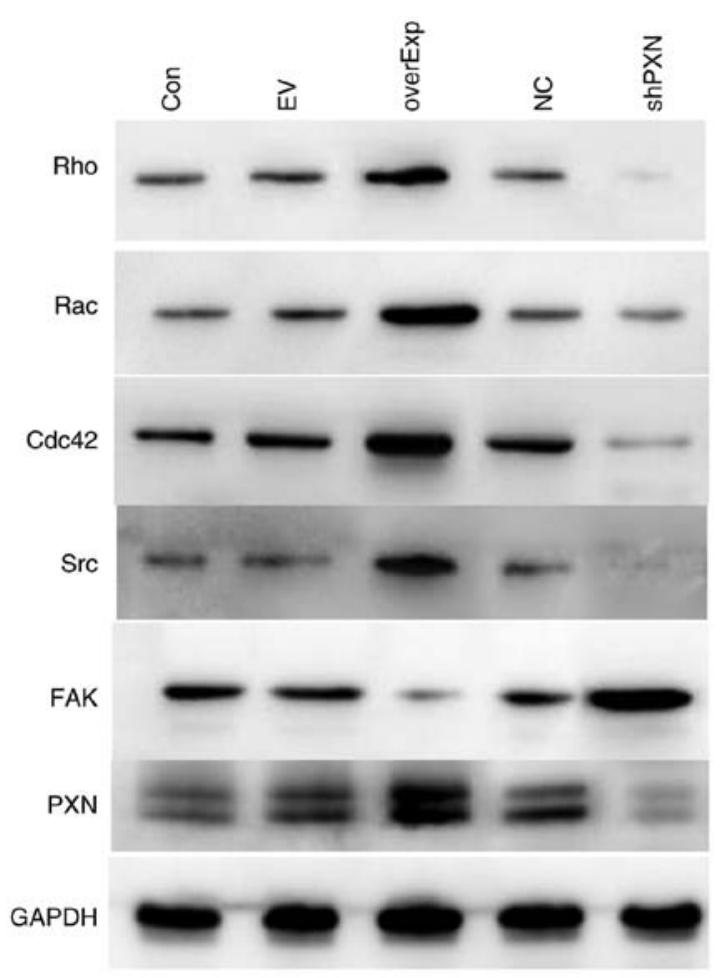

Figure 2. Protein expression levels of members of the PXN signaling pathway. Protein expression levels of Rac, Rho, Cdc42, Src and PXN were lower in the shPXN group and higher in the overEXP group. FAK expression levels were higher in the shPXN group. PXN, paxillin; Rac, AKT serine/threonine kinase 1; Cdc42, cell division control protein 42; Src, SRC proto-oncogene, non-receptor tyrosine kinase; Con, control group; EV, empty vector-transfected control group; shPXN, PXN knockdown group; NC, PXN-NC group; overExp, PXN over-expressed group.

Compared to the control group, the protein expression levels of Rac, Rho, Cdc42, Src and PXN were decreased in the shPXN group and increased in the overEXP group. Furthermore, FAK expression levels were increased in the shPXN group (Fig. 2).

shPXN decreases the migratory capacity of HUVECs. Migration of HUVEC was assessed using a Transwell assay. As shown in Fig. 3, compared with the empty vector-transfected control group, the number of cells which had migrated was significantly higher in the overexpression group $(\mathrm{P}<0.05$; Fig. 3F). Compared with the PXN-NC group, migration in the shPXN group was significantly lower $(\mathrm{P}<0.05$; Fig. 3F).

PXN enhances tube formation in HUVECs. Tube formation was detected in HUVECs. As shown in Fig. 4, tube formation was significantly increased in the overexpression group compared with the empty vector-transfected control group $(\mathrm{P}<0.05$; Fig. 4F). Furthermore, tube formation was significantly decreased in the shPXN group compared with the PXN-NC group $(\mathrm{P}<0.05$; Fig. 4F).

PXN regulates a series of genes in vivo. PXN protein expression levels were determined by western blotting. The levels of Rac, Rho, Cdc42, Src and PXN were lower in the shPXN group compared with the model group, and higher in the overEXP group ( $\mathrm{P}<0.05$; Fig. 5). Additionally, FAK expression levels were higher in the shPXN group. 

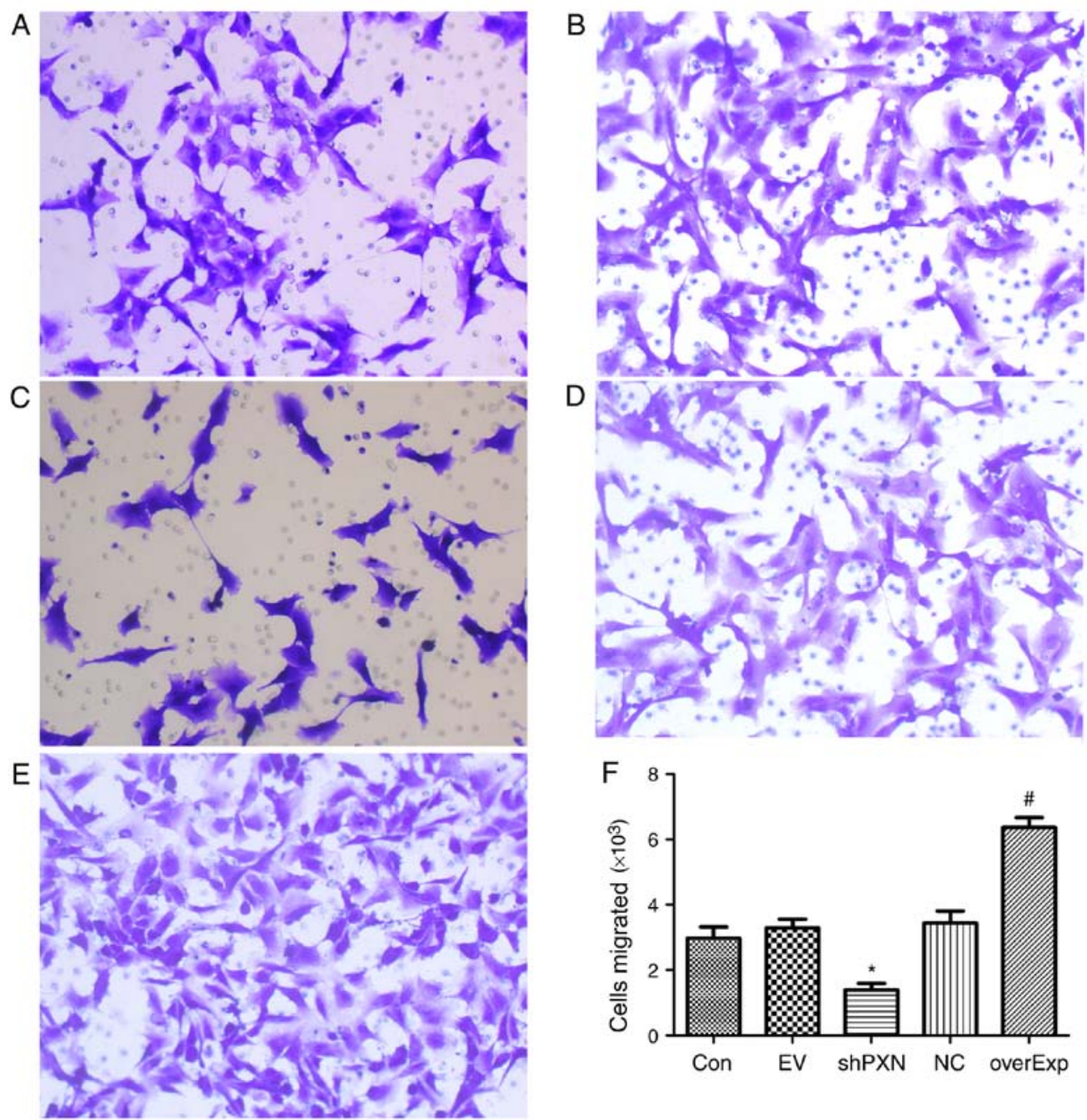

Figure 3. Migration of transfected HUVECs. Representative images of Transwell migration in (A) control, (B) empty vector-transfected, (C) shPXN, (D) PXN-NC and (E) PXN overexpression group. (F) Quantification of migration. "P $<0.05$ vs. EV and " $\mathrm{P}<0.05$ vs. NC. Con, control group; EV, empty vector-transfected control group; shPXN, PXN knockdown group; NC, PXN-NC group; overExp, PXN over-expressed group.

Expression of VEGF in vivo. Expression of VEGF was significantly higher in the model group compared with the control group ( $\mathrm{P}<0.05$; Fig. 6). Compared with the control group, VEGF expression was also significantly lower in the shPXN group and significantly higher in the overEXP group.

Angiogenesis in vivo. Capillaries could be seen growing from the corneal limbus to the corneal stroma in the model and overexpression groups (Fig. 7). No notable differences were observed between the PXN knockdown and the control groups.

\section{Discussion}

Corneal avascularity is important for maintaining corneal transparency and ensuring good eyesight. However, a number of corneal diseases, such as infection, trauma and chemical injury, can cause neovascularization to spread from the limbal cornea to the corneal endothelium. This neovascularization will undermine the transparency of the cornea and alter the physiological microenvironment, causing damage to eyesight and even blindness (13). According to records, $4 \%$ of ophthalmology patients in the United States are affected by corneal neovascularization (14). Neovascularization causes a variety of ophthalmological diseases and eventual blindness, and has thus become a major issue in the field of ophthalmology (15). At present, there is still a lack of effective treatments for corneal neovascularization $(15,16)$. The product of the PXN gene is a cytoskeletal phosphoric acid protein, primarily located in focal adhesions $(17,18)$. PXN is a complex protein that was first discovered in $\mathrm{v}$-Src-transfected cells (19), though its role in neovascularization remains unclear and controversial. Shortly thereafter, purified protein was obtained from smooth muscle tissue, and was identified as a focal adhesion binding protein (20). PXN was proposed to function in the uptake of muscle active proteins and at sites of cell adhesion. Although PXN itself lacks enzymatic activity, it contains a variety of structural domains that can bind signaling and structural proteins $(21,22)$. Therefore, PXN has been proposed to be a cytoskeletal protein that can effectively transmit signals (23-26). Research has shown that paxillin mediates the adhesion, migration, proliferation and cytoskeletal attachment of cells. Our previous study demonstrated that PXN promotes VEGF-A-induced proliferation, migration, adhesion and capillary formation in HUVECs (9). 

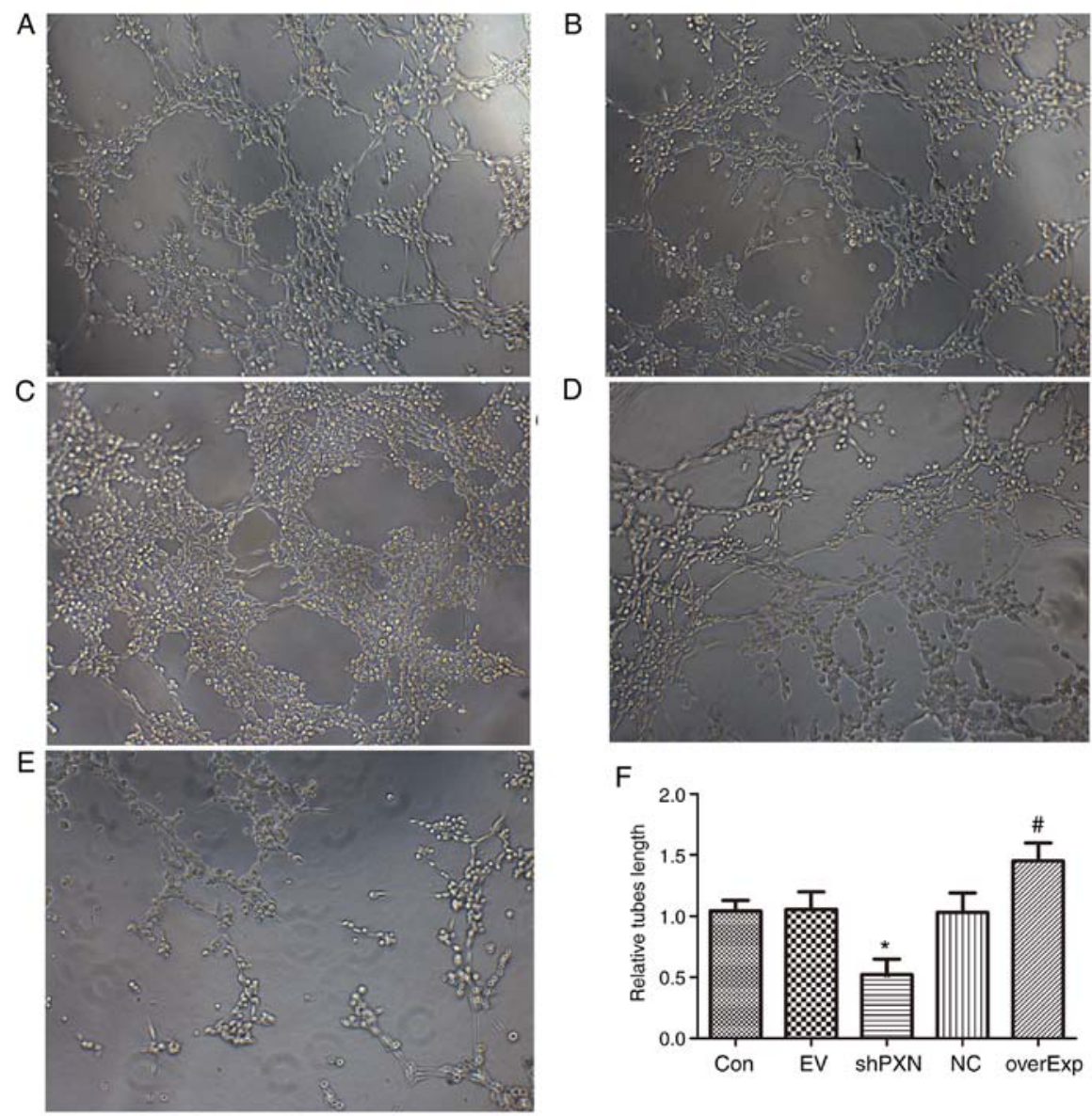

D
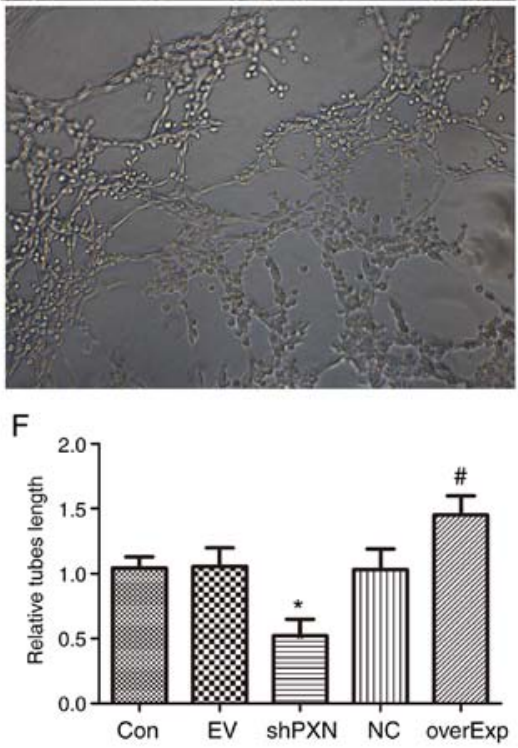

Figure 4. Tube formation in HUVECs. Representative images of tube formation in (A) control, (B) empty vector-transfected, (C) shPXN, (D) PXN-NC and (E) PXN overexpression group. (F) Quantification of tube formation. " $\mathrm{P}<0.05$ vs. EV and ${ }^{~} \mathrm{P}<0.05$, vs. NC. Con, control group; EV, empty vector-transfected control group; shPXN, PXN knockdown group; NC, PXN-NC group; overExp, PXN over-expressed group.

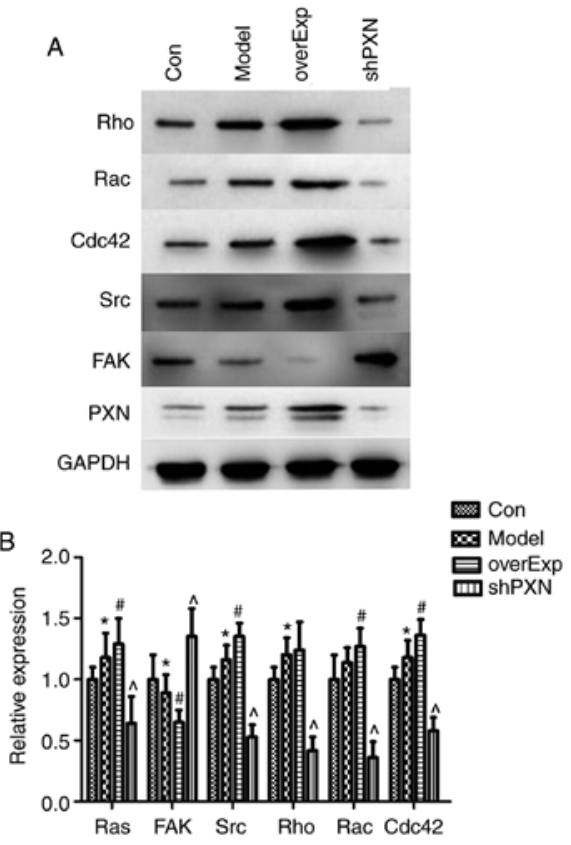

Figure 5. PXN regulates a series of genes in vivo. (A) Representative western blots of Rho, Rac, Cdc42, Src, FAK and PXN. (B) Expression of Rac, Rho, Cdc42, Src, and PXN was lower in the shPXN group and higher in the overEXP group. FAK levels were higher in the shPXN group. " $\mathrm{P}<0.05$ vs. Con ${ }^{\text {" }} \mathrm{P}<0.05$ vs. model and ${ }^{\wedge} \mathrm{P}<0.05$ vs. overExp. Con, control group; Model, model group; overExp, PXN over-expressed group; shPXN, PXN knockdown group.
Minamiguchi et al (27) reported that PXN is essential for neovascularization in tumor formation. Previous studies have shown that PXN also serves an important role in corneal neovascularization $(8,9,28,29)$. In the present study, HUVECs and a mouse corneal neovascularization model were used to determine changes in PXN-mediated signaling during corneal neovascularization induced by inflammation.

The establishment of adhesion junctions between endothelial cells is a key component of angiogenesis (30). Formation of an endothelial network is the premise of both initial angiogenesis as well as regeneration of blood vessels. Node formation between endothelial cells is key to various types of angiogenesis (31). Cell migration involves dynamic spatial changes in the cytoskeleton and cell attachment (32). Rho, $\mathrm{Rac}$, and Cdc 42 are members of the GTP binding protein Rho subfamily (Rho-GTPase). Rho-GTPases serve an important role in cell migration by regulating the rearrangement of the cytoskeleton and stress fiber formation (33-35). Rac and Rho regulate the aggregation of agonist proteins, and promote the production of plate pseudopods and stress fibers in migrating cells (36). Cdc 42 promotes the production of filamentous pseudopods and regulates the direction of migration (37). Rho can also increase the activity of myosin light chain kinase, phosphorylate the myosin light chain, induce an increase in stress fibers and promote cell migration (38). Rac regulates phosphorylation of the myosin light chain, increases the tension of 

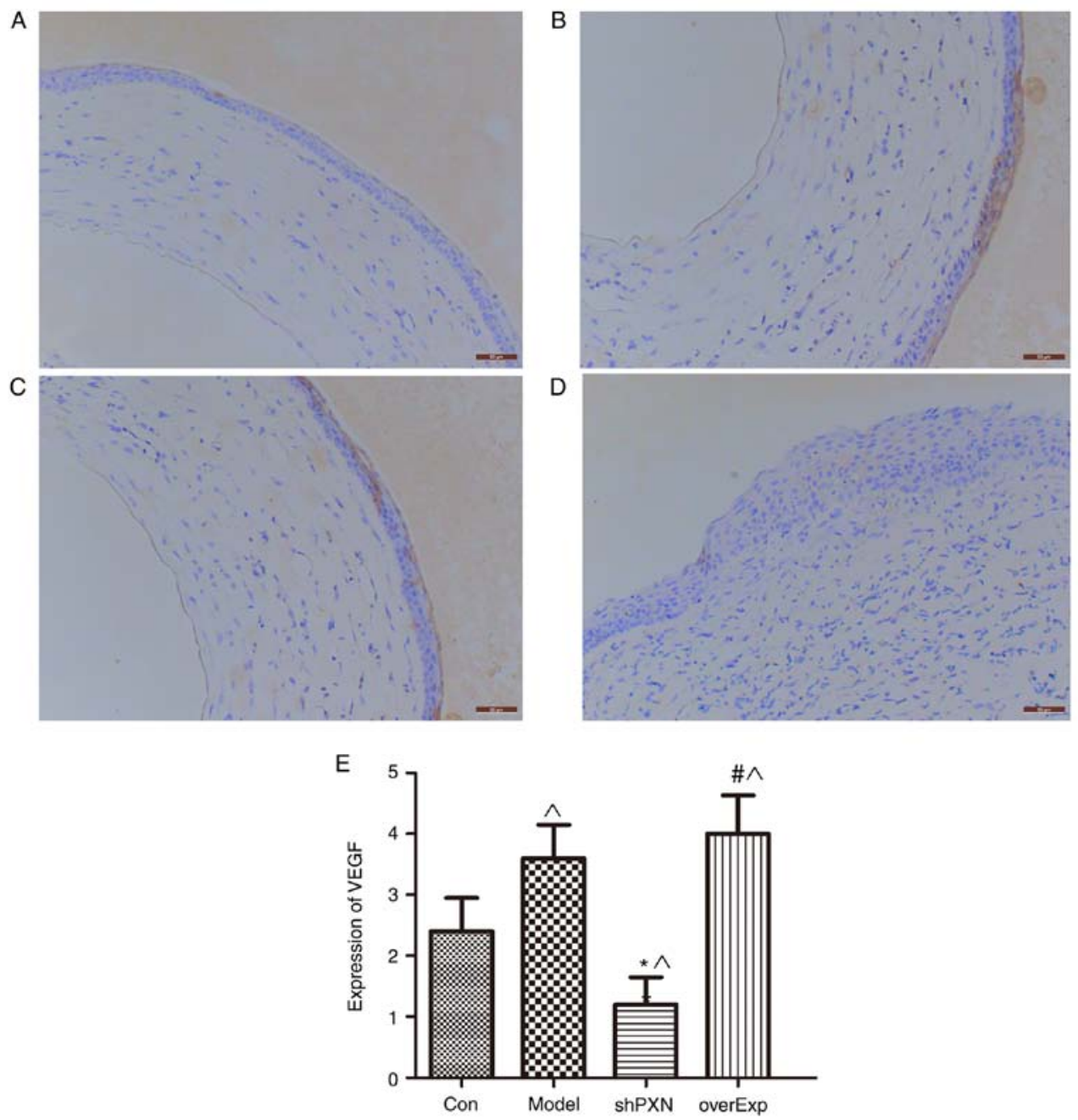

Figure 6. VEGF expression was detected using immunohistochemistry. Representative immunohistochemical slides of (A) the control group, (B) model group, (C) PXN knockdown group and (D) PXN over-expression group. (E) Expression of VEGF was significantly higher in the model group compared with the control group. Expression of VEGF was lower in the shPXN group and higher in the overEXP group. ${ }^{\wedge} \mathrm{P}<0.05$ vs. Con; ${ }^{*} \mathrm{P}<0.05$ vs. model and ${ }^{\sharp} \mathrm{P}<0.05$ vs. shPXN group. VEGF, vascular endothelial growth factor; Con, control group; shPXN, PXN knockdown group; overExp, PXN over-expressed group.
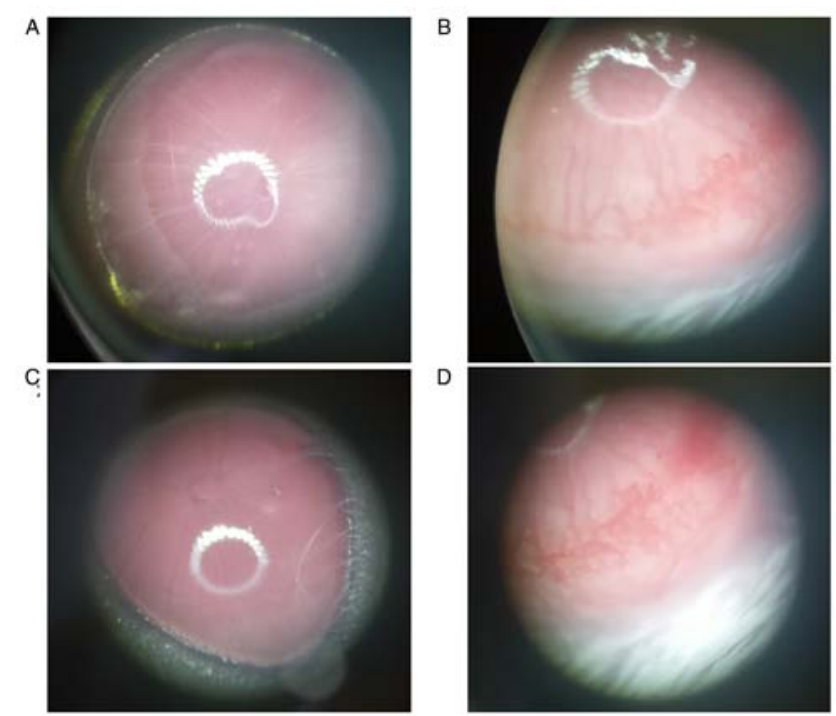

Figure 7. Representative images of (A) control group, (B) model group, (C) PXN knockdown group and (D) over expression group. Capillaries were seen growing from the corneal limbus to the corneal stroma in the model and overexpression groups. There was no significance difference between the PXN knockdown and the control group. the cytoskeleton and regulates cell contraction, which is the basis of focal adhesion and stress fibers (38). Furthermore, $\mathrm{Cdc} 42$ and Rac cooperate to regulate endothelial cell lumen formation during vascular regeneration (39). In the present study, the levels of Rac, Rho, Cdc42 and PXN protein were lower in the shPXN group and higher in the overEXP group, suggesting that PXN downregulates Rac, Rho and Cdc42, and promotes angiogenesis and cell migration. This is consistent with the cell migration results, given the involvement of Rac, Rho and Cdc42 in migration.

PXN is controlled by a series of genes, including Ras, FAK, Src, GIT, PIX, NCK, PT538, PKC- $\delta$, RAC, MEKK1 and MKK4/7, both FAK and Src can modulate the phosphorylation status of paxillin at Tyr31 and Tyr118 (40). In the present study, it was demonstrated that FAK promoted PXN expression, whereas Src inhibited it. These results are consistent with those of Sachdev et al (41) in which FAK was shown to promote the phosphorylation of PXN, and increase the expression of Rho and Rac, whilst inhibiting Cdc42. In the present study, compared with the empty vector-transfected control group, cell migration was significantly increased in the overexpression group. Therefore, PXN promotes the migration 
of HUVECs. Lisiak et al (7) reached a similar conclusion, where it was demonstrated that downregulation of PXN inhibited migration of human breast cancer cells. Furthermore, German et al $(8)$ and Sero et al $(28,29)$ showed that a decrease in PXN expression enhanced the migration of HUVECs. In addition, PXN has been shown to serve a key role in tumor progression. Findings from the present study showed that tube formation was significantly increased in HUVECs and mice in the overexpression group, and thus PXN may facilitate angiogenesis.

In summary, the present study showed that during corneal angiogenesis, PXN drove the migration of endothelial cells and promoted angiogenesis. However, the detailed underlying mechanism remains unknown. The results highlight the role of PXN in corneal angiogenesis, and suggest that targeting the PXN signaling pathway may inhibit corneal angiogenesis, providing a theoretical basis for the prevention of corneal diseases.

\section{Acknowledgements}

The authors would like to pay their respects to ZMM, who died in the fight against COVID-19 on 3rd March 2020.

\section{Funding}

The present study was supported by grants from the National Natural Science Foundation Youth Project (grant no. 81800802) and the Wuhan Municipal Health and Family Planning Committee Guidance Project (Wuhan, China; grant no. WX17Z02).

\section{Availability of data and materials}

All data generated or analyzed during this study are included in the published article.

\section{Authors' contributions}

WJY and YQX designed the study and wrote the manuscript. JBY, WY, YX, LZ, YNY, ZMM and FZ performed the experiments. YX performed the statistical analysis. All authors read and approved the final version of the manuscript.

\section{Ethics approval and consent to participate}

All experimental protocols were approved by the Institutional Animal Care and Use Committee of Tongji Medical of Huazhong University of Science and Technology and Animal Experimental Ethical Inspection of Laboratory Animal Centre, Huazhong Agriculture University (approval no. HZAUMO-2017-039).

\section{Patient consent for publication}

Not applicable.

\section{Competing interests}

The authors declare that they have no competing interests.

\section{References}

1. Gong X and Rubin LP: Role of macular xanthophylls in prevention of common neovascular retinopathies: Retinopathy of prematurity and diabetic retinopathy. Arch Biochem Biophys 572: 40-48, 2015.

2. Hackett SF, Seidel C, Abraham S, Chadha R, Fortmann SD, Campochiaro PA and Cooke JP: The nicotinic cholinergic pathway contributes to retinal neovascularization in a mouse model of retinopathy of prematurity. Invest Ophthalmol Vis Sci 58: 1296-1303, 2017.

3. Choudhry N, Golding $\mathrm{J}$ and Rao RC: Vitreous invasion: Neovascular frond in proliferative diabetic retinopathy. Ophthalmology 123: 2625, 2016.

4. Nguyen QD, De Falco S, Behar-Cohen F, Lam WC, Li X, Reichhart N, Ricci F, Pluim J and Li WW: Placental growth factor and its potential role in diabetic retinopathy and other ocular neovascular diseases. Acta Ophthalmol 96: e1-e9, 2018.

5. Singh NK, Kotla S, Kumar R and Rao GN: Cyclic AMP response element binding protein mediates pathological retinal neovascularization via modulating DLL4-NOTCH1 signaling. EBioMedicine 2: 1767-1784, 2015.

6. Nakama T, Yoshida S, Ishikawa K, Kubo Y, Kobayashi Y,Zhou Y, Nakao S, Hisatomi T, Ikeda Y, Takao K, et al: Therapeutic effect of novel single-stranded RNAi agent targeting periostin in eyes with retinal neovascularization. Mol Ther Nucleic Acids 6: 279-289, 2017.

7. Lisiak N, Paszel-Jaworska A, Toton E, Rubis B, Pakula M, Bednarczyk-Cwynar B, Zaprutko L and Rybczynska M: Semisynthetic oleanane triterpenoids inhibit migration and invasion of human breast cancer cells through downregulated expression of the ITGB1/PTK2/PXN pathway. Chem Biol Interact 268: 136-147, 2017.

8. German AE, Mammoto T, Jiang E, Ingber DE and Mammoto A: Paxillin controls endothelial cell migration and tumor angiogenesis by altering neuropilin 2 expression. J Cell Sci 127: 1672-1683, 2014

9. Yang WJ, Yang YN, Cao J, Man ZH, Li Y and Xing YQ: Paxillin regulates vascular endothelial growth factor A-induced in vitro angiogenesis of human umbilical vein endothelial cells. Mol Med Rep 11: 1784-1792, 2015.

10. Livak KJ and Schmittgen TD: Analysis of relative gene expression data using real-time quantitative PCR and the 2(-Delta Delta C(T)) method. Methods 25: 402-408, 2001.

11. Watanabe M, Da Fonseca CD and Vattimo Mde F: Instrumental and ethical aspects of experimental research with animal models. Rev Esc Enferm USP 48: 181-188, 2014.

12. Morton DB and Griffiths PH: Guidelines on the recognition of pain, distress and discomfort in experimental animals and hypothesis for assessment. Vet Rec 116: 431-436, 1985.

13. Voiculescu OB, Voinea LM and Alexandrescu C: Corneal neovascularization and biological therapy. J Med Life 8: 444-448, 2015.

14. Norooznezhad AH, Norooznezhad F and Ahmadi K: Next target of tranilast: Inhibition of corneal neovascularization. Med Hypotheses 82: 700-702, 2014.

15. Hsu CC, Chang HM, Lin TC, Hung KH, Chien KH, Chen SY, Chen SN and Chen YT: Corneal neovascularization and contemporary antiangiogenic therapeutics. J Chin Med Assoc 78: 323-330, 2015.

16. Gupta D and Illingworth C: Treatments for corneal neovascularization: A review. Cornea 30: 927-938, 2011.

17. Nikolopoulos SN and Turner CE: Actopaxin, a new focal adhesion protein that binds paxillin LD motifs and actin and regulates cell adhesion. J Cell Biol 151: 1435-1448, 2000.

18. Kratimenos P, Koutroulis I, Marconi D, Syriopoulou V, Delivoria-Papadopoulos M, Chrousos GP and Theocharis S: Multi-targeted molecular therapeutic approach in aggressive neuroblastoma: The effect of Focal Adhesion Kinase-Src-Paxillin system. Expert Opin Ther Targets 18: 1395-1406, 2014.

19. Grgurevich S, Mikhael A and Mcvicar DW: The Csk homologous kinase, Chk, binds tyrosine phosphorylated Paxillin in Human Blastic T cells. Biochem Biophys Res Commun 256: 668-675, 1999.

20. Brown MC, Perrotta JA and Turner CE: Identification of LIM3 as the principal determinant of paxillin focal adhesion localization and characterization of a novel motif on paxillin directing vinculin and focal adhesion kinase binding. J Cell Biol 135: 1109-1123, 1996 
21. Tsai WC, Yu TY, Lin LP, Lin MS, Tsai TT and Pang JS: Platelet rich plasma promotes skeletal muscle cell migration in association with up-regulation of FAK, paxillin, and F-Actin formation. J Orthop Res 35: 2506-2512, 2017.

22. Wu KH, Ho CT, Chen ZF, Chen LC, Whang-Peng J, Lin TN and Ho YS: The apple polyphenol phloretin inhibits breast cancer cell migration and proliferation via inhibition of signals by type 2 glucose transporter. J Food Drug Anal 26: 221-231, 2018.

23. Jagadeeswaran R, Zumba O, Yala S and Salgia R: Paxillin and MET interactions promote lung cancer growth, invasion, and angiogenesis. Cancer Res: 68, 2008.

24. Lyck R, Reiss Y, Gerwin N, Greenwood J, Adamson P and Engelhardt B: T-cell interaction with ICAM-1/ICAM-2 double-deficient brain endothelium in vitro: The cytoplasmic tail of endothelial ICAM-1 is necessary for transendothelial migration of T cells. Blood 102: 3675-3683, 2003.

25. Stiegler AL, Draheim KM,Li X, Chayen NE, Calderwood DA and Boggon TJ: Structural basis for paxillin binding and focal adhesion targeting of $\beta$-parvin. J Biol Chem 287: 32566-32577, 2012.

26. Turner CE: Paxillin and focal adhesion signalling. Nat Cell Biol 2: E231-E236, 2000

27. Minamiguchi K, Kumagai H, Masuda T, Kawada M, Ishizuka M and Takeuchi T: Thiolutin, an inhibitor of HUVEC adhesion to vitronectin, reduces paxillin in HUVECs and suppresses tumor cell-induced angiogenesis. Int J Cancer 93: 307-316, 2001.

28. Sero JE, German AE, Mammoto A and Ingber DE: Paxillin controls directional cell motility in response to physical cues. Cell Adh Migr 6: 502-508, 2012.

29. Sero JE, Thodeti CK, Mammoto A, Bakal C, Thomas S and Ingber DE: Paxillin mediates sensing of physical cues and regulates directional cell motility by controlling lamellipodia positioning. PLoS One 6: e28303, 2011.

30. Lampugnani MG and Dejana E: Adherens junctions in endothelial cells regulate vessel maintenance and angiogenesis. Thromb Res 120 (Suppl 2): S1-S6, 2007.

31. De Souza Junior DA, Mazucato VM, Santana AC, Oliver C and Jamur MC: Mast cells interact with endothelial cells to accelerate in vitro angiogenesis. Int J Mol Sci 18: 2674-2679, 2017.

32. Ridley AJ, Allen WE, Peppelenbosch M and Jones GE: Rho family proteins and cell migration. Biochem Soc Symp 65 111-123, 1999.
33. Krause-Gruszczynska M, Rohde M, Hartig R, Genth $H$, Schmidt G, Keo T, Konig W, Miller WG, Konkel ME, Konkel ME and Backert S: Role of the small Rho GTPases Racl and Cdc42 in host cell invasion of Campylobacter jejuni. Cell Microbiol 9: 2431-2444, 2007

34. Hernández-Garcia R, Iruela-Arispe ML, Reyes-Cruz G and Vazquez-Prado J: Endothelial RhoGEFs: A systematic analysis of their expression profiles in VEGF-stimulated and tumor endothelial cells. Vascul Pharmacol 74: 60-72, 2015.

35. Kusuhara S, Fukushima Y, Fukuhara S, Jakt LM, Okada M, Shimizu Y, Hata M, Nishida K, Negi A, Hirashima M, et al: Arhgef15 promotes retinal angiogenesis by mediating VEGF-induced Cdc42 activation and potentiating RhoJ inactivation in endothelial cells. PLoS One 7: e45858, 2012.

36. Nobes CD and Hall A: Rho, rac, and cdc42 GTPases regulate the assembly of multimolecular focal complexes associated with actin stress fibers, lamellipodia, and filopodia. Cell 81: 53-62, 1995.

37. Nakashima M and Lazo JS: Phosphatase of regenerating liver-1 promotes cell migration and invasion and regulates filamentous actin dynamics. J Pharmacol Exp Ther 334: 627-633, 2010.

38. Brzeska H, Szczepanowska J, Matsumura F and Korn ED: Rac-induced increase of phosphorylation of myosin regulatory light chain in HeLa cells. Cell Motil Cytoskeleton 58: 186-199, 2010.

39. Bayless KJ and Davis GE: The Cdc42 and Rac1 GTPases are required for capillary lumen formation in three-dimensional extracellular matrices. J Cell Sci 115: 1123-1136, 2002.

40. Vindis C, Teli T, Cerretti DP, Turner CE and Huynh-Do U: EphB1-mediated cell migration requires the phosphorylation of paxillin at Tyr-31/Tyr-118. J Biol Chem 279: 27965-27970, 2004.

41. Sachdev S, Bu Y and Gelman IH: Paxillin-Y118 phosphorylation contributes to the control of Src-induced anchorage-independent growth by FAK and adhesion. BMC Cancer 9: 12, 2009.

(i) (i) () This work is licensed under a Creative Commons

EY NG NO Attribution-NonCommercial-NoDerivatives 4.0 International (CC BY-NC-ND 4.0) License. 